\title{
DETECTION AND INVESTIGATION OF ANOMALOUS (UNDAMPED) THERMAL WAVES
}

\author{
Vladimir I. Vysotskii, Anton O. Vasilenko, Mikhail V. Vysotskyy
}

Shevchenko Kiev National University, http://www.univ.kiev.ua

64/13, st. Vladimirskaya, 01601 Kyiv, Ukraine

vivysotskii@gmail.com, vivysotskii@gmail.com, mihas1984@gmail.com

\section{Alla A. Kornilova, Efim I. Hait, Nailia H. Volkova}

Lomonosov Moscow State University, http://www.msu.ru

1/2, Leninskie Gory, 119991 Moscow, Russian Federation

prfnart@mail.ru,haitov5@yandex.ru,haitov5@yandex.ru

Abstract. Results of the detection and study of fundamentally new physical phenomenon - generating undamped temperature waves, which can exist in different environments only at certain frequencies, the value of which depends on the time of the local relaxation of thermal excitations in these environments (the thermalization time) are presented. In the air, under normal conditions, the minimal frequency of such wave corresponds to $70-90 \mathrm{MHz}$ and in metals and semiconductors it is $10^{12}-10^{14} \mathrm{~Hz}$. In the experiments these waves are generated via cavitation processes and registered in the air at a distance of $2 \mathrm{~m}$ and this distance is limited only by the experimental conditions

Keywords: cavitation, shock waves, acoustic detector, thermal wave, the heat equation, thermal relaxation

PACS: 47.55.Bx; 43.25.Yw; 43.25.Ed; 44.10.+i; 62.50.Ef; 66.70.-f

Bibliography - 22 references

Received 03.12.2016

RENSIT, 2016, 8(2):196-206

DOI: $10.17725 /$ rensit.2016.08.196

\section{Contents}

1. INTRODUCTION (196)

2. The THERMAL DIFFUSIVITY EQUATION AND ITS SOLUTIONS FOR SYSTEMS WITH MEMORY (197)

3. Registration AND STUDY OF UNDAMPED TEMPERATURE WAVES IN CAVITATION EXPERIMENTS (199)

4. Conclusion (203)

REFERENCES (205)

\section{INTRODUCTION}

In our previous studies [1-6] various radiative processes that accompany the cavitation of liquid jet exiting under pressure from a narrow channel were considered. At the time of these studies we have observed non-trivial physical processes during the interaction of this jet with the target. In particular the excitation of shock waves in this target and generation of pulsed X-ray and high-frequency (in the range $70-90 \mathrm{MHz}$ ) radiations from the opposite surface of the target have been observed.
High-frequency radiation were recorded by an acoustic sensor at a distance of $2 \mathrm{~m}$ from the target. This fact is paradoxical since it is well known that the absorption coefficient of hypersound in air in normal conditions is

$\delta(\omega) \approx 10^{-12} \omega^{2} \mathrm{sm}^{-1}$

and in this frequency range is equal to a very large value $\delta(\omega) \approx 10^{4} \mathrm{~cm}^{-1}$, while the path length does not exceed a few microns. In addition as a sensor wideband acoustic detector with a resonant frequency of 1-2 $\mathrm{MHz}$ was used, which sensitivity in the hypersound range $\omega>>1 \mathrm{MHz}$ is low. Herewith we had taken every precaution to make it impossible any path of signal from source to detector, except the direct propagation in the air. Further analysis has shown that the registered radiation is not the hypersound, but the thermal (temperature) radiation, which has a non-damped character. The physical mechanism of excitation and propagation of such waves is discussed below. 


\section{THE THERMAL DIFFUSIVITY EQUATION AND ITS SOLUTIONS FOR SYSTEMS WITH MEMORY}

Let's consider the background, rationale, and "weak" places of the classical heat equation, the solution of which leads to the thermal waves.

For decades in the analysis of heat conduction problem the classical Fourier hypothesis was used, according to which the non-stationary heat flow $\vec{q}(\vec{r}, t)$ is proportional to the temperature gradient $\vec{q}(\vec{r}, t)=-\lambda \operatorname{grad}(T(\vec{r}, t))$,

and extends in the direction of reduction of this gradient (eg., [7-9]). Here, $\lambda$ is the coefficient of thermal conductivity.

If we combine (2) with the energy conservation law for the local area (the continuity equation) in a medium with a volume density $\rho$ and heat capacity $\mathrm{c}_{\mathrm{v}}$

$$
\rho c_{v} \frac{\partial T(\vec{r}, t)}{\partial t}=\operatorname{div} \vec{q}(\vec{r}, t),
$$

we can get a classic parabolic equation for the temperature field

$$
\rho c_{v} \frac{\partial T(\vec{r}, t)}{\partial t}=\lambda \operatorname{div}\{\operatorname{grad}[T(\vec{r}, t)]\} .
$$

In the one-dimensional case the solution of this equation is a superposition of colliding plane waves

$$
\begin{aligned}
& T(\omega, x, t)=A_{\omega} e^{i(\omega t-k x)}+B_{\omega} e^{i(\omega t+k x)} \equiv \\
& \equiv A_{\omega} e^{-\delta x} e^{i(\omega t-\kappa x)}+B_{\omega} e^{\delta x} e^{i(\omega t+\kappa x)}, \\
& k=\kappa(1-i), \kappa=\operatorname{Re} k=\sqrt{\omega / 2 G}, \delta=\operatorname{Im} k=\sqrt{\omega / 2 G} .
\end{aligned}
$$

Here, $G=\lambda / \rho c_{v}-$ thermal diffusivity coefficient

This solution shows that the temperature waves, produced on the basis of the system of equations (2-4) are characterized by a very strong damping with a factor $\delta=\sqrt{\omega / 2 G}$, which is exactly equal to the wave number $\kappa$.

It is obvious that the solution (5) with such ratio of wave number and the absorption coefficient at which virtually complete absorption of wave occurs on a spatial interval of the wavelength can be called wave only with great approximation.

Such "standard" solutions are well known. They, in particular, are regarded as illustrations in any textbook on mathematical physics and are used for solving of some applications (in particular, the problem of propagation of extremely low-frequency temperature wave in soil, which is caused by seasonal temperature variations with a period of 1 year). Analysis of the initial equations (2)-(4) shows that at least two important conditions are used there: the principle of local thermodynamic equilibrium and the principle of locality. The principle of locality allows to use the energy conservation equation in differential (local) form instead of the conservation of energy equation in integral form. The principle of local thermodynamic equilibrium justifies the possibility of describing of non-equilibrium system, in which there is a temperature gradient, concentration gradient, etc., through the introduction of local equilibrium states of smaller subsystems. These very important principles are valid only for slow processes, when the relaxation time of subsystems to equilibrium state $\tau$ is much shorter than the characteristic time of the process (eg, the duration of the heat front for pulse impact or oscillation period for harmonic thermal effects).

In the 50 s of the 20 th century in the works of Cattaneo and Vernotte attempts to consider non-stationary heat transfer processes have been made, which has led to the hyperbolic equation for the temperature field $[10,11]$. Hyperbolic and nonlinear parabolic models of heat transfer for the purpose of the analysis of the new heat transfer modes, including the mode with escalation were also considered [12]. Unfortunately, these and similar "incomplete" methods and models do not allow to use the thermal processes for the efficient processing of the experimental data, related to the interaction of fast particles with the environment (for example, crystalline targets).

This issue is discussed in details in our works [13-16]. Methods discussed below show that more correct and adequate is the revision of the basic ratios and initial concepts, which are used without limits of obtaining the "standard" initial relations (2-4). The main of them relates to the hypothesis of the use of locally equilibrium (thermalized) environment for the receiving of these relations. To make this circumstance correct we need to evaluate a real time of thermal relaxation in different environments [17, 18]. 
In plasma the time of formation of equilibrium (Maxwellian) distribution within the electronic subsystem in a small area equals

$$
\tau^{(e e)} \approx \sqrt{m_{e}}\left(k_{B} T_{e}\right)^{3 / 2} / 4 \pi \Lambda n_{e} e^{4}
$$

where $n_{\mathrm{e}}, m_{\mathrm{e}}, T_{\mathrm{e}}-$ respectively, the electron concentration, mass of electron and electron temperature, $\Lambda \approx 15$ - "coulomb" logarithm.

For the ionic subsystem in plasma the relaxation time equals $\tau^{(i i)} \approx \sqrt{m_{i} / m_{e}} \tau^{(e e)}$.

Close to this value relaxation time, which determines the "maxwellization" of electron gas on the levels of energy in the conduction band, adjacent to the Fermi level, approximately equals $\tau \approx 10^{-14}-10^{-12}$ seconds.

In the air the relaxation time $\tau \approx(1.5-2) \cdot 10^{-8} \mathrm{sec}$ is defined as by the duration of "maxwellization" process of gas translational degrees of freedom $\tau_{\operatorname{maxv}}$ as by the much longer relaxation time of molecules vibrational and rotational degrees of freedom. With the change of temperature and, especially, density and composition of the air (e.g., at the presence of water vapor), the value of $\tau$ can vary within a wide range $\left(\tau \approx 10^{-7}-10^{-8} \mathrm{~s}\right)$.

It is also evident that the relaxation time has to depend on the intensity of the thermal wave and on the temperature of medium in which the wave propagates. The solution of such nonlinear problem is an extremely difficult problem.

It is obvious that for thermal processes, which occurr slowly than the given values $\tau$, taking into account of relaxation is negligible because for the description of such processes it is always possible to use equilibrium characteristics.

The simplest account of the temperature relaxation process (without nonlocality and time heterogeneity of the process of temperature formation) can be carried out using a modified equation of continuity

$$
\rho c_{v} \frac{\partial T(\vec{r}, t+\tau)}{\partial t}=-\operatorname{div} \vec{q}(\vec{r}, t),
$$

corresponding to the integral relation

$$
\frac{\partial}{\partial t} \int_{V} W_{T}(\vec{r}, t+\tau) d V=-\int_{S} \vec{q}(\vec{r}, t) \vec{n} d S,
$$

which has been obtained by integration of the differential relation (7a) using the Gauss formula.
Here $W_{T}(\vec{r}, t+\tau)=\rho c_{v} T(\vec{r}, t+\tau)$ - the volume density of the thermal energy; $\vec{n}$ - normal vector to the surrounding the volume $V$ surface.

Formula (7b) shows that the change in the total heat energy at time $t+\tau$ in the small volume $V$ is determined by the energy flow $\vec{q}(\vec{r}, t)$ via the surface $S$, the bounding this volume, corresponding to the previous point of time $t$.

Substituting equation (2) to (7a), we obtain the thermal diffusivity equation with delay $\tau$, which is in one-dimensional case in a homogeneous medium has the form

$$
\frac{\partial T(x, t+\tau)}{\partial t}=G \frac{\partial^{2} T(x, t)}{\partial x^{2}} \text {. }
$$

The solution of equation (8) is determined by the superposition of temperature waves

$$
\begin{aligned}
& T(\omega, x, t)=A_{\omega} \exp \left(-\kappa \frac{\cos \omega \tau}{\sqrt{1+\sin \omega \tau}} x\right) \exp \{i(\omega t-\kappa \sqrt{1+\sin \omega \tau} x)\}+ \\
& +B_{\omega} \exp \left(\kappa \frac{\cos \omega \tau}{\sqrt{1+\sin \omega \tau}} x\right) \exp \{i(\omega t+\kappa \sqrt{1+\sin \omega \tau} x)\},
\end{aligned}
$$

$\cos \omega \tau \geq 0, \kappa=\sqrt{\omega / 2 G}$,

obtained for the first time in [13-16]. Each of these waves is fundamentally different from the temperature waves, corresponding to "standard" solution (5).

For waves determined by solution (9), the damping coefficient

$$
\delta=\kappa \frac{\cos \omega \tau}{\sqrt{1+\sin \omega \tau}}=\sqrt{\omega / 2 G} \frac{\cos \omega \tau}{\sqrt{1+\sin \omega \tau}}
$$

depends both on the coefficient thermal diffusivity $G=\lambda / \varrho c_{v}$, so and on the time of delay $\tau$ and the wave frequency $\omega$. At extremely low values of $\omega \tau<<1$ from (10a), we can find

$\delta \approx \sqrt{\omega / 2 G}(1-\omega \tau / 2)$.

At $\tau=0$ the solutions (5) and (10a,b) coincide.

In Figure 1 the frequency dependences of the absorption coefficient of the "conventional" thermal waves in a medium with no delay (at $\tau=0)$ and the same wave with a delay $(\tau \neq 0)(10)$ are presented. The absorption coefficient has a physical sense only when $\delta / \kappa \geq 0$ (in the upper half-plane of Fig. 1). For graphics (1) the value of $\tau$ is a formal parameter, which is needed for comparison of (1) and (2) within the same scale.

It can be seen that for an arbitrary value $\tau$ the absorption coefficient of thermal waves in an 


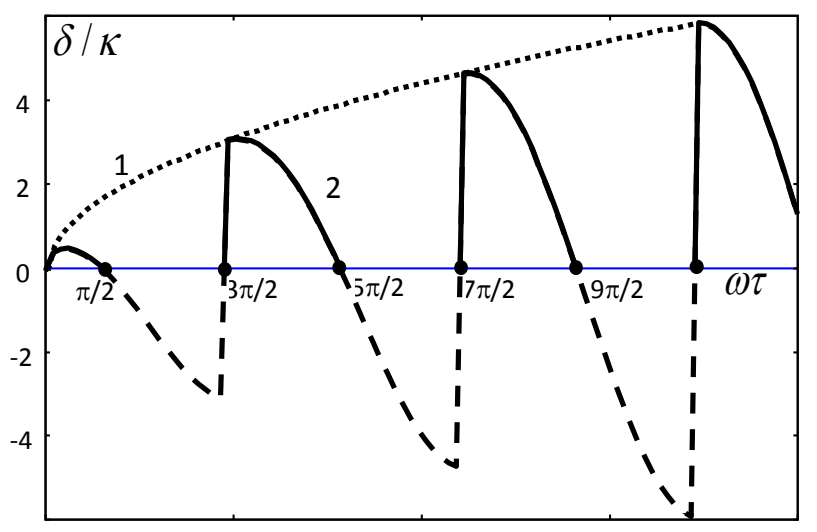

Fig. 1. The frequency dependence of the normalized absorption coefficient of the temperature wave: 1 - a solution (5) of the "classical" thermal diffusivity equation (4), corresponding to $\tau=$ $0 ; 2$ - solution (10) of the thermal diffusivity equation (8) with an arbitrary delay $\tau$.

environment with a delay $\tau \neq 0$ is always less than a medium without such delay. From the analysis of the solution (9) follows that heat waves can exist only if $\cos \omega \tau \geq 0$, which corresponds to the physical result - the wave in the medium is damped in the propagation direction. Excitation of temperature waves with a frequency corresponding to the condition $\cos \omega \tau<0$ in the equation (9) is impossible, since the existence of such waves is contrary to the principle of causality - their amplitude increases in the propagation direction.

To the waves with frequencies

$\omega_{\mathrm{n}}=(n+1 / 2) \pi / \tau, n=0,1,2 \ldots$

which correspond to conditions $\cos \omega_{\mathrm{n}} \tau=0$, wave numbers $k_{n}= \pm \kappa_{n} \sqrt{2}$ are real and damping coefficient $\delta(10 a)$ is zero, and the general solution of equation (9) in this case has the form of a superposition of the direct and inverse undamped temperature waves

$$
\begin{aligned}
& T\left(\omega_{n}, x, t\right)=A_{\omega_{n}} \exp \left\{i\left(\omega_{n} t-\kappa_{n} \sqrt{2} x\right)\right\}+ \\
& +B_{\omega_{n}} \exp \left\{i\left(\omega_{n} t+\kappa_{n} \sqrt{2} x\right)\right\}, \\
& \kappa_{n}=\sqrt{\omega_{n} / 2 G} .
\end{aligned}
$$

The physical mechanism of formation of these waves is connected with the influence of the thermal relaxation on phase conditions, which determine the energy dissipation of the thermal motion.

Excitation of these waves is possible in different ways.
The most optimal one is connected with a possible use of local periodic heating at a frequency (11), which correspond to one of such waves. Such system can be based, for example, on the process of interaction of high-frequency electromagnetic wave with a frequency $\omega_{n}$ with an absorbing surface. Such surface is a source of undamped thermal waves. It is also possible to use waves with a carrier frequency essentially higher than $\omega_{n}$ (e.g., laser radiation), which are modulated by this frequency.

Another simpler case is connected with action on the border between the air and condensed matter by short acoustic pulses with duration $\Delta t$ less than the time $\tau$ of relaxation of thermal excitation in the air. In this case in the spectrum of the local thermal waves generated in the area of the local heating, which is formed by the action of these pulses, non-absorbing thermal waves with frequencies corresponding to the condition (11) are present. Such case is realized in the experiments discussed below.

\section{REGISTRATION AND STUDY OF UNDAMPED TEMPERATURE WAVES IN CAVITATION EXPERIMENTS}

The research of the propagation in the air of waves, which are not electromagnetic or acoustic, and the formation of which was stimulated on the target surface by very short mechanical shocks, connected with cavitation phenomena in liquids, was carried out on the basis of several systems used by us during the experiments on cavitation water jets $[1,4,5,17,18]$.

The sceme of such system is shown in Figure 2.

In this system water under pressure of $250 \mathrm{~atm}$ was pumped through channel and through nozzle of small diameter into a cavitation chamber. At the output of the channel cavitation cloud of bubbles appeared, which was formed by the process of growth of cavitation bubbles in a moving jet water. The action of this jet on the inner surface of the metal target caused the formation of intensive shock waves. The inner surface of metal target is at a distance of $14 \mathrm{~mm}$ from the channel outlet 
opening, while the space between this surface and the nozzle formed the cavitation chamber.

For the registration of thermal waves in the air on the outer border of the metal target broadband acoustic piezoceramic receiver PZT-19 with a diameter of $20 \mathrm{~mm}$ and a resonant frequency of $1 \mathrm{MHz}$ was used.

In first experiments the receiver moved along the installation axis in the interval from $5 \mathrm{~mm}$ to $21 \mathrm{~cm}$ from the outer surface of the metal target. Measurements were made every $1 \mathrm{~cm}$. For control measurements the receiver was turned by 0,20 , 90 and 180 degrees relative to the axis of the experimental setup. In the experiments tungsten and molybdenum targets were used.

Figure 3 shows the form of signal, detected by the receiver at different distances from a tungsten target. It can be seen that in the space behind the target (in the air) both low-frequency (LF) and high frequency (HF) signals were recorded, whose frequencies changed with the distance weakly.

A simple analysis shows that low-frequency signal with a frequency $\omega_{\mathrm{LF}} \approx 8.3-8.9 \mathrm{kHz}$ corresponds to a spatial acoustic resonance inside the cavitation chamber. This resonance correspond to "positive feedback loop" during the sequential motion of the shock wave from the target through the walls of cavitation chamber to the nozzle channel and corresponding modulation parameters of this nozzle, affecting the speed of formation of cavitation bubbles in the jet water. This resonance contributed to the formation of the regime of periodic synchronized cavitation of jet liquid, in which the cavitation bubbles are arise

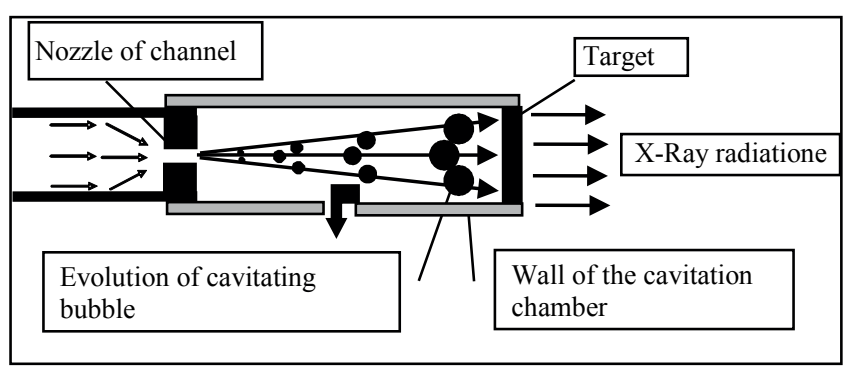

and formed not sporadically, but as synchronized groups.

Such system is similar to the classical electron Van der Pol generator. A small variation of this frequency can occur due to a reverse action of low-frequency acoustic waves reflected from the receiver towards the target.

The amplitude of the low-frequency signal by removing the receiver from the target decreases and is characterized by an approximate coefficient of effective wave attenuation $\delta_{\mathrm{LF}} \approx 0.05 \mathrm{~cm}^{-1}$. Such damping agrees good enough with the laws of acoustics and is determined by the combined action of two factors: a) "usual" linear damping of low-frequency sound wave; b) diffractive extension of the low-frequency wave front, which is generated by a source of limited aperture.

Fundamentally different situation corresponds to the process of registration of the HF signal, the amplitude of which increases with the increasing of distance of HF wave at removal from the target synchronously with a decrease of amplitude of the LF wave! These results are presented in Figure 4.

A possible explanation for this surprising phenomenon may be connected with a hypothetical transfer of heat energy from one wave to another (damping and relaxation of low-frequency thermal excitation leads to the generation of highfrequency thermal wave). The mechanism of this process has not been determined.

At great distances from the target the saturation of amplitude is observed. The maximum value of the amplitude of this wave corresponds to the distance of $21 \mathrm{~cm}$. It is necessary to point

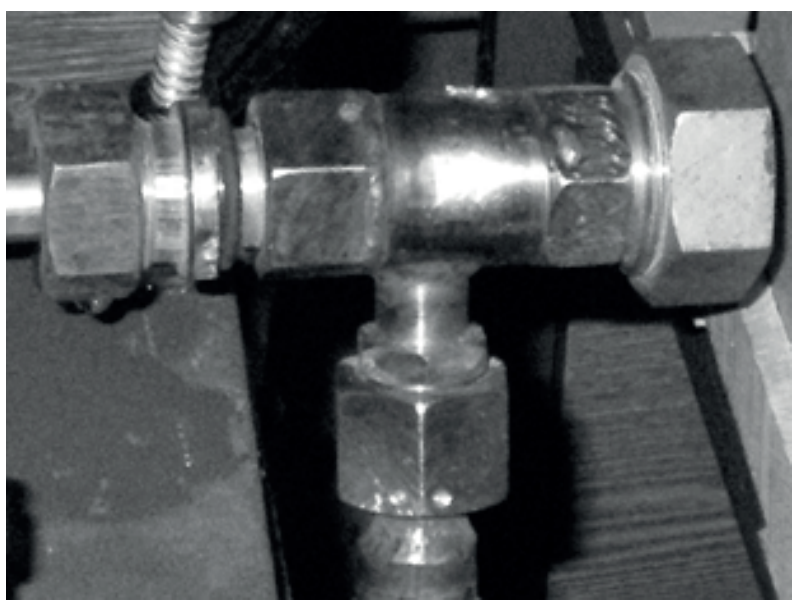

Fig. 2. Schematic picture and photo of setup for generating of X-rays at cavitation of water jet in closed chamber. 
out that both signals (both low frequency and high frequency) decreased in amplitude when the receiver was turned in vertical and horizontal planes. It proves that signals were emitted by the target and they were not the background.

It should be noted that the registration of the acoustic signal with a frequency of to $\sim 80-85 \mathrm{MHz}$
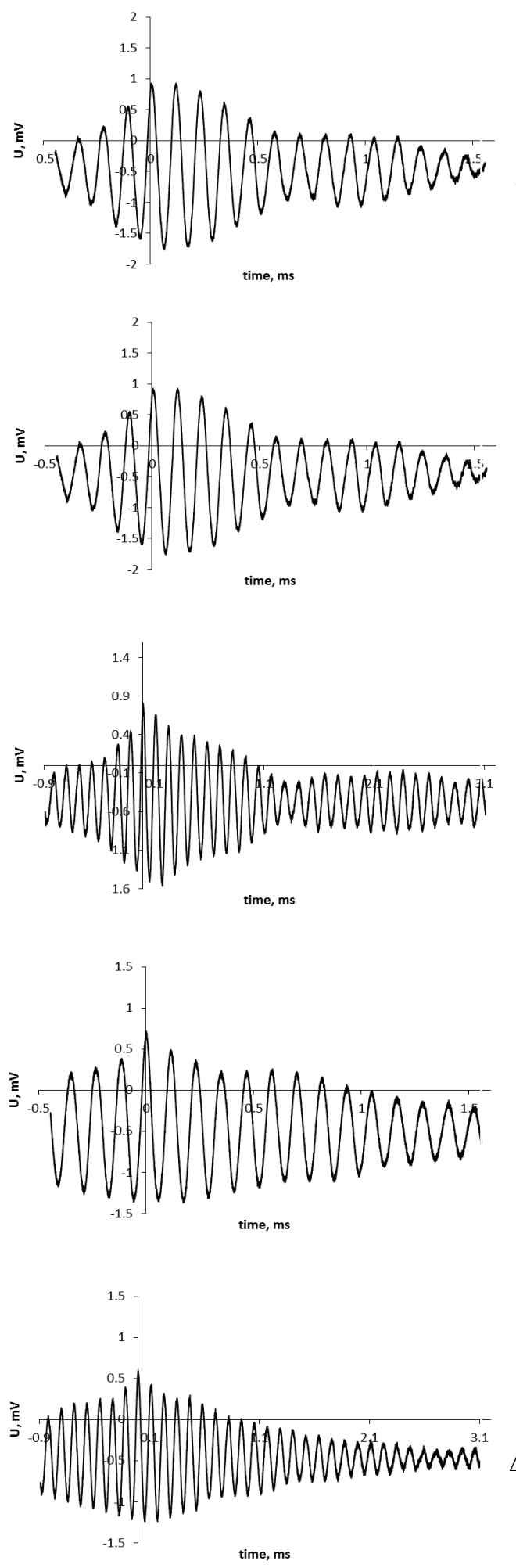
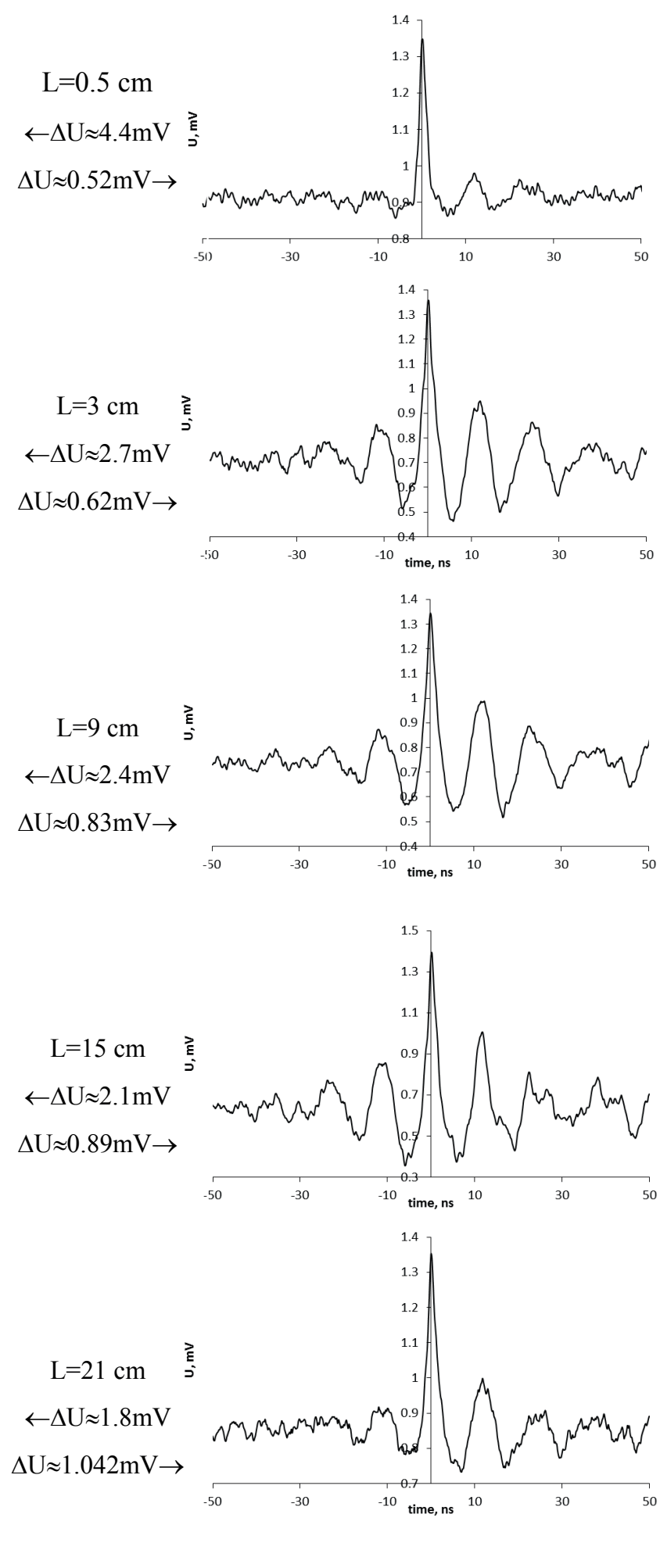

Fig. 3. Low-frequency (left, $\omega_{L F} \approx 8.3-8.9 \mathrm{kHz}$ ) and high-frequency (right, $\omega_{H F} \approx 80-85 \mathrm{MHz}$ ) signals recorded by an acoustic receiver, which is located in the air at different distances L from the target. The amplitudes of the recorded signals $\Delta U$ are presented near the respective plots. 


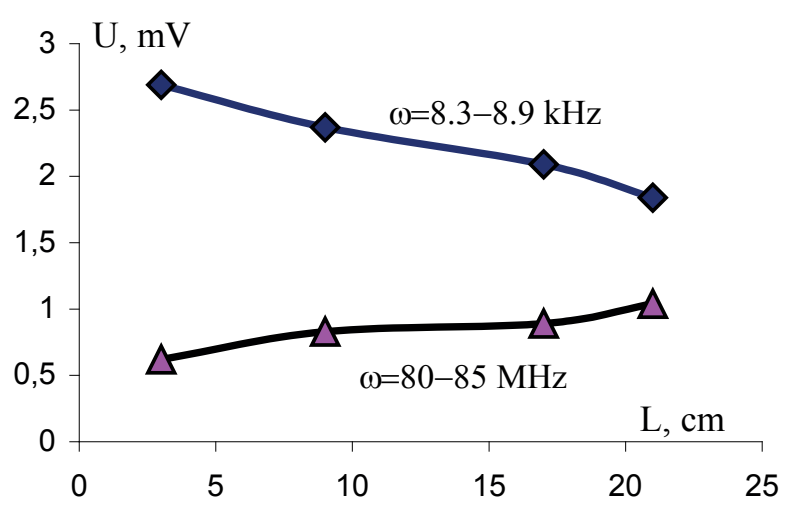

Fig. 4. Synchronous change amplitudes of low and high frequency thermal waves in the air when it changes of the distance from the source to the place of registration.

at a distance of $L=10-20 \mathrm{~cm}$ from the outer surface of the target (opposite to the direction of water jet falling in the state of cavitation) is a paradox which cannot be explained on the basis of "standard" acoustics. This conclusion follows directly from the expression for sound attenuation coefficient for gases and liquids (eg., [19])

$$
\delta(T)=\frac{\omega^{2}}{2 \rho\{c(T)\}^{3}}\left[\left(\frac{4}{3} \eta+\xi\right)+\lambda(T)\left(\frac{1}{c_{V}}-\frac{1}{c_{P}}\right)\right],
$$

where $c(T) \approx\left(331.3+1.21 T^{\circ}\right) \cdot 10^{2} \mathrm{~cm} / \mathrm{sec}-$ the speed of sound, $\varrho-$ air density, $c_{\mathrm{p}}=1000(\mathrm{~J} / \mathrm{kg} \cdot \mathrm{K})$ and $c_{\mathrm{v}}=717(\mathrm{~J} / \mathrm{kg} \cdot \mathrm{K})$ - specific heat capacity of air at room temperature and normal pressure at constant pressure and volume, $\eta=1.9 \cdot 10^{-5}(\mathrm{~Pa} \cdot \mathrm{sec})$ and $\xi=17.2 \cdot 10^{-6}(\mathrm{~Pa} \cdot \mathrm{sec})$ - coefficients of the shear and bulk viscosity of air, $T^{\circ}-$ the temperature in Celsius.

From this formula follows that the hypersound with the frequency $\omega \approx 80-85 \mathrm{MHz}$ propagation in the air at room temperature corresponds to a very large damping coefficient $\delta(T) \approx 10000 \mathrm{~cm}^{-1}$. Path lenght of such wave in the air is less than $<>\approx 1-2 \mu \mathrm{m}$, which is in 10000 times (!) less than the experimental results.

This result becomes even more paradoxical taking into account that the resonant frequency of the acoustic detector used was $1 \mathrm{MHz}$, which is 85 times lower than the frequency of the detected signal and indicates a rather low detection efficiency. So it is evident that the real amplitude of HF signal in the location of acoustic detector was very great.
The second paradox is connected with the discussed above effect of spatial increas of amplitude of the HF wave, which is synchronized with a corresponding decrease of the amplitude of the LF wave.

These paradoxes can be solved if we assume that the HF wave is not the acoustic wave but the undamped temperature wave, which discussed above.

The frequency of this wave is in good agreement with results of calculation.

In the air the relaxation time is defined as by the duration of the process of "maxwellisation" $\tau_{\max v}$, as by longer processes of internal and mutual relaxation of vibrational and rotational states of $\mathrm{N}_{2}$ and $\mathrm{O}_{2}$ molecules. The value $t_{\max }$ depends on the average cross-section $\sigma \approx \sqrt{2} \pi D^{2}$ of elastic scattering of similar size molecules $\mathrm{N}_{2}$ and $\mathrm{O}_{2}$ (their diameter can be taken as $D \approx 3 \AA$ ), which are basic molecules in the air, on the average velocity of gas molecules $v=\sqrt{3 k T / m} \approx 4.6 \cdot 10^{4} \mathrm{~cm} / \mathrm{s}$ and the total concentration of molecules $n \approx 3 \cdot 10^{19} \mathrm{~cm}^{-3}$. Under normal conditions (normal pressure and room temperature) we can find

$$
\tau_{\max v} \approx 10 / n<\sigma(v) v>\approx 10 \sqrt{m / 3 k T} / n<\sigma>\approx 2 n s .
$$

Accounting of the relaxation of the rotational and vibrational states of molecules allows to characterize the time of local relaxation $\tau \approx 15-20 n s$, which corresponds to the evaluation of the minimum frequency of undamped temperature wave in the range of $\omega_{0} \approx 70-90 \mathrm{MHz}$ and varies with variations of pressure and humidity.

Another series of research has been carried out using similar experimental equipment, based on the installation of the KMT setup, considered in [17, 18]. The schematic diagram of this setup is similar to setup presented in Fig. 2, and the difference is only in the direction of free outlet of water jet. The purpose of this research was to examine the possibility of extending the temperature waves at substantially greater distances.

Figure $\mathbf{5}$ shows a fragment of panoramic spectrum of registered waves in frequency range up to $300 \mathrm{MHz}$ when $L=18.5 \mathrm{~cm}$, as well as fragments of registered signal at a distance $L=18.5 \mathrm{~cm}$ and $L=198 \mathrm{~cm}$ from the place of 

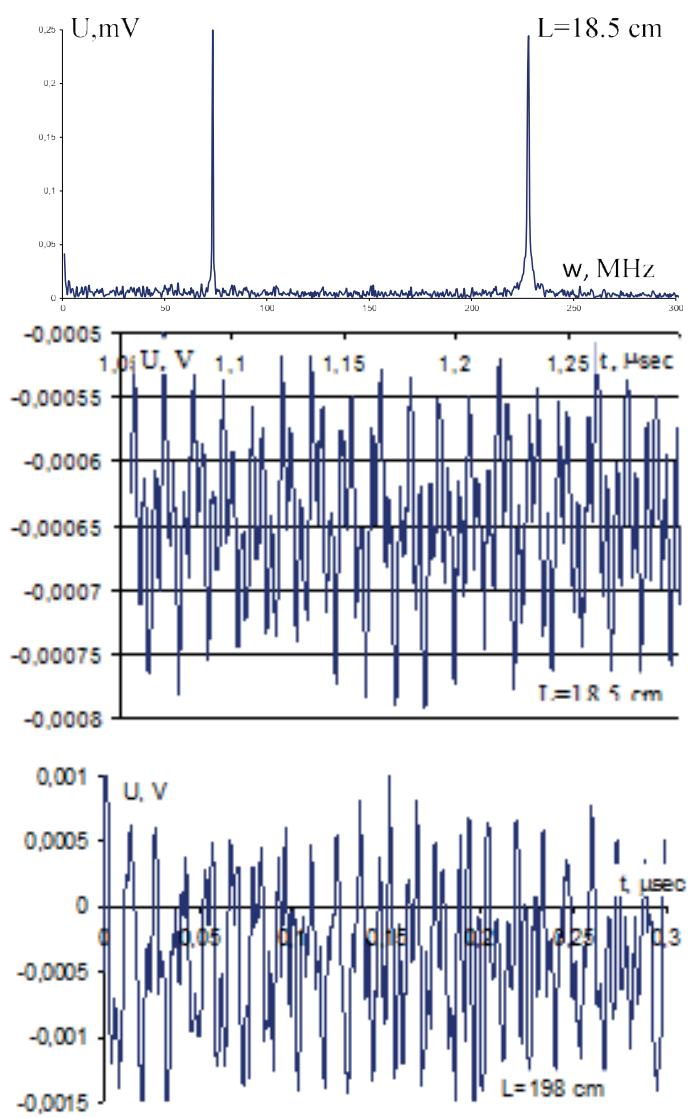

Fig. 5. Part panoramic spectrum signals recorded by acoustic detector that located at a distance $L=18.5 \mathrm{~cm}$ from the outer surface of the target, and the fragments detected signal at a distance $L=18.5 \mathrm{~cm}$ and $L=198 \mathrm{~cm}$.

formation of these waves (the outer surface of the tube, through which a water jet passed). A similar panoramic spectrum was registered and at other distances from the emitting surface $(0.5,18.5,100$ and $198 \mathrm{~cm}$ ).

The frequencies of the signals registered by the acoustic detector with a resonant frequency of $1 \mathrm{MHz}$, are respectively $\omega_{0} \approx 74 \mathrm{MHz}$ and $\omega_{1} \approx 225 \mathrm{MHz}$, and are in good agreement with the formula (11), which determines the spectrum of undamped temperature waves. The difference between the lowest frequency of these waves from the frequency $\omega \approx 80-85 \mathrm{MHz}$, registered in the previous experiment, can be connected with the fact that these frequencies are determined by the thermal relaxation time (14), which varies with the density, temperature of air and air composition Since in this experiment a jet of water after the generation of shock waves came out into the free space, it has led to high humidity in the space around the experimental setup and, as a result, could change a little relaxation time (from $\tau=\pi \omega_{0} / 2 \approx 18.4-19.6$ ns at $\omega_{0} \approx 80-85 \mathrm{MHz}$ to $\tau=\pi \omega_{0} / 2 \approx 21$ ns at $\omega_{0} \approx 74 \mathrm{MHz}$ ). It is obvious that similar reason (in particular, a small change of air temperature near the experimental equipment, which has worked for a long time of the experiment that lasted several hours with concomitant rearrangement and fixing of the detector at different distances, and a corresponding change in concentration of molecules) explains a slight difference of wave frequencies $\omega_{0} \approx 80-85 \mathrm{MHz}$ registered at different distances.

Despite the fact that these waves are detected at substantially different distances from the place of formation ( $L=18.5 \mathrm{~cm}$ and $L=198 \mathrm{~cm}$ ), their amplitudes at the output of the registration system were identical and were equal to $U_{\max } \approx 1 \mathrm{mV}$, that confirms the absence of their damping in the air.

\section{CONCLUSION}

Conducted experiments demonstrate the registering of unknown before our work phenomenon - the generation and propagation of undamped HF thermal (temperature) waves with frequency $\omega_{0} \geq 75-85 \mathrm{MHz}$, which are registered by acoustic detector at a great distance (up to 2 meters) from the source.

It should be noted that this distance is limited only by the size of the laboratory, and it is expected that such waves will propagate without attenuation at a much greater distances. These waves are fundamentally different from the "classic" hypersonic waves of megahertz range, which can not propagated in the air and are damped at a very small distance (a units and tens of microns) from the place of excitation.

These undamped thermal waves can be excited and propagated only in environments with a finite (non-zero) time of the local thermodynamic relaxation and their frequency is determined completely by this time.

It was shown that the frequency of these waves depends on the propagation environment parameters (in this case - the air). Condition of excitation of such waves is connected with the obvious requirement that in the spectrum of thermal excitation the spectral components at these frequencies were present. This condition 
limits the parameters of thermal pulses - their duration should be small enough so that the needed frequencies were presented in the spectrum.

Used method of excitation of thermal waves by shock waves of cavitation satisfies these conditions because the minimum duration of the leading edge of shock wave is equal $\Delta t_{\min } \approx \Delta l / \mathrm{v}_{\text {Swe }}$, where $\Delta l \approx 10 d$ the length of the shock wave front, which is determined by the average distance $d$ between atoms or molecules, $\mathrm{v}_{\mathrm{SW}}-$ the speed of the shock wave, that is defined by the Mach number and the speed of sound in this medium.

In metals and other condensed media $\mathrm{v}_{\mathrm{SW}} \approx(2-5) \cdot 10^{5} \mathrm{~cm} / \mathrm{s}, d \approx(1.5-2) \AA$ and $\Delta t_{\min } \approx$ $(0.3-0.8) \cdot 10^{-13}$ s. So in the air at normal pressure $\mathrm{v}_{\mathrm{SW}} \approx(0.5-1) \cdot 10^{5} \mathrm{~cm} / \mathrm{s}, d \approx n_{\text {air }}^{-1 / 3} \approx 30 \AA$ and $\Delta t_{(\text {air) } \min } \approx 10^{-12} \mathrm{~s}$.

From these estimations follow that under optimal conditions corresponding to the formation of shock waves the maximum frequency of undamped thermal waves in the air can reach $\omega_{\max } \approx 1 / \Delta t \approx 1 \mathrm{GHz}$. This estimation is in very good agreement with the results of the experiment discussed above. In Figure $\mathbf{6}$ a high-frequency part of the general panorama of the spectrum of registered thermal waves is presented, the lowfrequency part of it was shown above in Fig. 5.

From the form of this spectrum follows that the maximum frequency of the undamped thermal waves at this method of excitation reaches $\omega_{\max } \approx 1 \mathrm{GHz}$, which coincides with the results of a theoretical estimations.

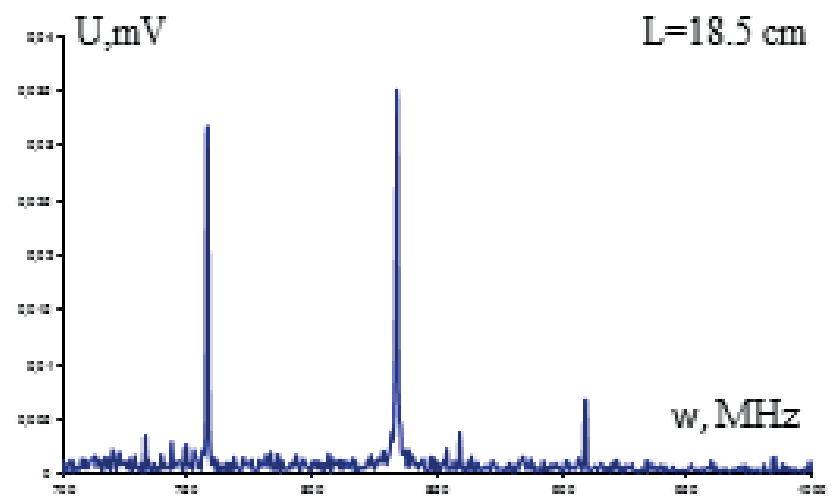

Fig. 6. The high-frequency part of the panoramic spectrum of signals recorded by acoustic detector that located at a distance $L=18.5 \mathrm{~cm}$ from the outer surface of the target.
These waves have a very small speed of propagation, which is according to estimates much smaller than the speed of normal sound and does not exceed $50-100 \mathrm{~m} / \mathrm{s}$.

Finally it is possible to note one more potentially possible factor of pulse generation of thermal waves at the output (outer) surface of screen or tube the inner surface of which is affected by acoustic shock waves caused by cavitation of jets of water. Earlier we considered the process of internal reflection of shock waves from the surface with the corresponding sharp "shaking" and the ionization of surface atoms as the main mechanism of excitation of thermal waves near the outer surface (the right surface of the target in Fig. 2), that leads to the generation of soft X-ray radiation and very rapid heating of the subsurface layer of air.

Recent results show that there is another mechanism of such pulsed heating, which is connected with peculiarities of shock pulse impact on atoms.

In [20-22], it was shown that at rapid (pulse) reversible deformation of the nonstationary harmonic oscillator the formation of coherent correlated states of particles takes place, when these particles are in nonstationary parabolic field of this oscillator. The peculiarities of this state leads to the synchronization of quantum fluctuations in the superposition state and to the formation of a giant fluctuations of momentum and kinetic energy, which are on many orders of magnitude greater than the average values. The result of this process is the sharp increase of the probability of chemical and nuclear reactions between particles in a parabolic field and particles that form this field, and pulsed energy release. In particular, in these works it have been shown that under such modulating action the fluctuations of kinetic energy of particles (in particular atomic nuclei) may reach $10-50 \mathrm{keV}$ at medium thermal (room) energy of target $\mathrm{kT} \approx 0.025 \mathrm{eV}$ !

Simple analysis shows that the process of shock wave motion through the condensed substance agrees with this scenario of intensified energy release. During the motion of the shock wave through such medium a strong compression 
of matter at its leading edge and the extension at the trailing edge takes place. For each atoms of the medium, which is surrounded by close neighbors, that is equivalent to the unsteady pulsed deformation of the harmonic oscillator, field of which is formed by nearest neighbors.

Such effect, which leads to the formation of coherent correlated states, plays the main role at the outside (output) surface of the cavitation chamber, where the reflection of shock waves from the border of dense material of the chamber and air takes place. Indirect confirmation of such mechanism of the energy release consist in the detection of foreign chemical elements on the outer chamber surface. These elements were absent before the exposure on the target with the water jet with cavitation bubbles. Such elements may be a result of pulsed nuclear reactions between the nuclei of the target and the nuclei of the atoms of the air. Such process is extremely short and its duration is determined by the time of existence of coherent correlated state [20-22].

We plan to explore this scenario doing additional and longer experiments.

\section{REFERENCE}

1. Vysotsky VI, KornilovaAA,Korneeva YuV,Krit TB. Issledovanie anomalnykh radiatsionnykh i teplovykh yavleniy pri kavitatsii strui zhidkosti. 1. Anomal'nye effekty pri generatsii rentgenovskogo izlucheniya, stimulirovannogo protsessom kavitatsii zhidkosti [The study of abnormal radiation and thermal phenomena in the cavitation jet fluid. 1. Anomalous effects in the generation of $\mathrm{X}$-rays, stimulate fluid cavitation]. Inzhenernaya firika, 2016, 2:33-45 (in Russ.).

2. Kornilova AA Vysotsky VI, Koldamasov AI, Hyun Ik Yang, Denis B. McConnell, Desyatov AB. Generation of intense directional radiation during the fast motion of a liquid jet through a narrow dielectric channel. Journal of surface investigation. X-ray, synchrotron and neutron techniques, 2007, 1(2):167-171.

3. Kornilova AA Vysotsky VI, Sysoev NN, Desyatov AB. Generation of X-rays at bubble cavitation in a fast liquid jet in dielectric channels. Journal of Surface Investigation. X-ray, Synchrotron and Neutron Techniques, 2009, 3(2):275-283.

4. Kornilova AA, Vysotsky VI, Sysoev NN, Litvin NK, Tomak VI, Barzov AA. Shockcavitational mechanism of X-ray generation during fast water stream cavitation. Moscow University Physics Bulletin, 2010, 65(1):46-50.

5. Kornilova AA Vysotsky VI, Sysoev NN, Litvin NK, Tomak VI, Barzov AA. Generation of intense $\mathrm{x}$-rays during ejection of a fast water jet from a metal channel to atmosphere. Journal of Surface Investigation. X-ray, Synchrotron and Neutron Techniques, 2010, 4(6):1008-1017.

6. Vysotsky VI, Kornilova AA, Sysoev NN. Rentgenovskoe izluchenie pri kavitatsii bystroy strui zhidkosti [X-rays at a fast cavitation liquid jet]. Radioelektronika. Nanosistemy. Informationnye tekbnologii (RENSIT), 2010, 2(1-2):57-69 (in Russ.).

7. Shashkoff AG, Bubnov VA, Janowski SYu. Volnovye yavleniya teploprovodnosti: sistemnostrukturny podkhod [Wave phenomena of thermal conductivity: system-structural approach]. Moscow, Editorial URSS, 2004, 296 p.

8. Telegin AS, Shwydkiy VS, Yaroshenko YuG. Teplomassoperenos [Heat and mass transfer]. Moscow, Akademkniga Publ., 2002.

9. Sobolev SL. Local non-equilibrium transport models. Physics Uspekhi, 1997, 40:1043-1053.

10. Cattaneo CR. Sur une forme de l'équation de la chaleur éliminant le paradoxe d'une propagation instantanée. Comptes Rendus, 1958, 247:431-433.

11. Vernotte P. Les paradoxes de la theorie continue de l'équation de la chaleur. Comptes Rendus, 1958, 246:3154-3155.

12. Samarsky AA, Galaktionov VA, Kurdyumov SP, Mikhaylov AP. Rezhimy s obostreniem [The sharpening regimes]. Moscow, Nauka Publ., 1987.

13. Vysotskii VI, Vassilenko VB, Vasylenko AO. Propagation of temperature waves in medium with internal thermal relaxation. Bulletin of Taras Shevchenko National Univ. of Kyiv, Series Rafiophysics, 2013, 1:11-14. 
14. Vysotskii VI, Vassilenko VB, Vasylenko AO. Periodic heat transfer regimes in active medium. Bulletin of Taras Shevchenko National Univ. of Kyiv, Series Physics \& Mathematics, 2013, 2:255-260.

15. Vasylenko AO, Vysotskii VI, Vassilenko VB. Heat transfer equation with delay for media with thermal memory. Intern. J. of Sciences: Basic and Applied Research (IJSBAR), 2015, 12(1):160-166.

16. Vysotskii VI, Vasilenko AO, Vassilenko VB, Vysotskyy MV. Nonequilibrium thermal effects during pulsed action on conducting medium. Inorganic Materials: Applied Research, 2015, 6(3):199-204.

17. Vysotskii VI, Kornilova AA, Vasilenko AO. Observation and investigation of $\mathrm{X}$-ray and thermal effects at cavitation. Current Science, 2015, 108(4):114-119.

18. Vysotsky VI, Kornilova AA, Vasilenko AO, Tomak VI. Detecting and investigation of undamped temperature waves excited under water jet cavitation. Surface Investigation. X-ray, Synchrotron and Neutron Techniques, 2014, 8(6):1086-1192.

19. Gurbatov SN, Rudenko OV. Akustika v zadachakh [Acoustics in problems]. Moscow, Nauka Publ., 1996.

20. Vysotskii VI, Vysotskyy MV. Coherent correlated states and low-energy nuclear reactions in non stationary systems. European Phys. Journal. A., 2013, 49(8):1-12.

21. Vysotskii VI, Adamenko SV, Vysotskyy MV. Acceleration of low energy nuclear reactions by formation of correlated states of interacting particles in dynamical systems. Annals of Nuclear energy, 2013, 62:618-625.

22. Vysotskii VI, Vysotskyy MV. The formation of correlated states and optimization of the tunnel effect for low-energy particles under nonmonochromatic and pulsed action on a potential barrier. Journal of Experimental and Theoretical Physics, 2015, 121 (4):559-571. 